\title{
Peripheral Brain Derived Neurotrophic Factor (BDNF) in Bulimia Nervosa: A Systematic Review
}

\author{
Kathryn Phillips \\ Fairfield University, kphillips1@fairfield.edu \\ Kathleen Keane \\ Barbara Wolfe
}

Follow this and additional works at: https://digitalcommons.fairfield.edu/nursing-facultypubs Copyright 2014 Elsevier/WB Saunders

The author post-print has been archived here with permission from the copyright holder under a Creative Commons Attribution Non-Commercial No Derivatives License.

\section{Repository Citation}

Phillips, Kathryn; Keane, Kathleen; and Wolfe, Barbara, "Peripheral Brain Derived Neurotrophic Factor (BDNF) in Bulimia Nervosa: A Systematic Review" (2014). Nursing and Health Studies Faculty Publications. 51.

https://digitalcommons.fairfield.edu/nursing-facultypubs/51

\section{Published Citation}

Phillips, Kathryn, Kathleen Keane, \& Barbara Wolfe. "Peripheral Brain Derived Neurotrophic Factor (BDNF) in Bulimia Nervosa: A Systematic Review", Archives of Psychiatric Nursing, 2014, 28(2):108-113. http://dx.doi.org/10.1016/ j.apnu.2013.11.006

This item has been accepted for inclusion in DigitalCommons@Fairfield by an authorized administrator of DigitalCommons@Fairfield. It is brought to you by DigitalCommons@Fairfield with permission from the rightsholder(s) and is protected by copyright and/or related rights. You are free to use this item in any way that is permitted by the copyright and related rights legislation that applies to your use. For other uses, you need to obtain permission from the rights-holder(s) directly, unless additional rights are indicated by a Creative Commons license in the record and/or on the work itself. For more information, please contact digitalcommons@fairfield.edu. 
Peripheral Brain Derived Neurotrophic Factor (BDNF) in Bulimia Nervosa: A Systematic Review

\author{
Kathryn Phillips, PhD, APRN \\ Kathleen Keane, MS, BSN, CNL, CCRN \\ Barbara E. Wolfe, PhD, RN, CS, FAAN
}

\title{
Introduction
}

Bulimia nervosa $(\mathrm{BN})$ is an eating disorder characterized by episodes of eating a large amount of food in a short period of time with a subjective experience of loss of control (American Psychiatric Association [APA], 2013). The caloric content resulting from the binge episodes is then compensated in some way such as self-induced vomiting or laxative misuse (APA, 2013). The onset of the disorder typically occurs during adolescence or young adulthood and affects more women than men (Hudson, Hiripi, Pope, \& Kessler, 2007). BN is associated with significant medical morbidity including electrolyte imbalances, dental erosion, and arrhythmias (Mehler, 2011). Currently there is limited understanding of the etiology of BN. Several biomarkers have been implicated in the etiology of BN, including brain derived neurotrophic factor (BDNF) (Nakazato, Hashimoto, Shimizu, Niitsu, \& Iyo, 2012). The purpose of this review is to examine investigations to date to further elucidate the possible role of BDNF in $\mathrm{BN}$.

\section{BDNF}

Compound characteristics. BDNF is a member of the neurotrophin family, a group of proteins with similar amino acid structures (Lewin \& Barde, 1996). The group includes nerve growth factor (NGF), the prototype, neurotrophin-3 (NT-3), neurotrophin-4 (NT-4/5) and BDNF 
(Lewin \& Barde, 1996). Neurotrophins selectively bind to one of the tyrosine kinase receptors

(Trk A, B, or C) and all neurotrophins bind to the tumor necrosis factor receptor p75NTR (Huang \& Reichardt, 2003). BDNF is widely distributed in the brain (Conner, Lauterborn, Yan, Gall, \& Varon, 1997), including areas associated with eating behavior (Bariohay, Lebrun, Moyse, \& Jean, 2005).

Synthesis. Chromosome 11 band p13 is the location of the gene that codes for BDNF (Maisonpierre et al., 1991). The creation of the BDNF protein begins with preproBDNF, a protein 240-260 amino acids long, which is formed from BDNF mRNA inside the endoplasmic reticulum of a cell (Lessman, Gottman, \& Malcangio, 2003). Prior to leaving the endoplasmic reticulum, the "pre" section is removed and the protein is sent as proBDNF to the Golgi apparatus for sorting (Lessman et al., 2003). The cell can release either proBDNF, the immature form of BDNF, or remove the "pro" section to release the mature form of BDNF (Lessman et al., 2003).

Circulation and measurement. BDNF freely crosses the blood brain barrier (Pan, Banks, Fasold, Bluth, \& Kastin, 1998). Pre-clinical evidence from mammalians found positive associations between brain levels of BDNF and that circulating in blood samples (Klein et al., 2011). A human study measuring BDNF in the internal jugular vein during exercise and at rest showed that the brain contributes approximately $72 \%$ of circulating BDNF at rest and $84 \%$ during exercise (Rasmussen et al., 2009). Other sources of circulating BDNF include muscles (Sakuma \& Yamaguchi, 2011), vascular endothelial cells (Nakahashi et al., 2000), and platelets (Fujimura et al., 2002).

Measurement of BDNF in the periphery can be done through serum or plasma blood samples using an enzyme-linked immunoassay (ELISA). Several studies provide evidence of 
reliability with little variability for BDNF measurement in serum or plasma with an ELISA (Elfving, Plougmann, \& Wegener, 2010; Trajkovska et al., 2007). While serum and plasma samples both provide reliable ELISA measures, serum samples of BDNF are estimated to be higher than levels in plasma, which may be due to the release of BDNF from platelets (Fujimura et al., 2002; Rosenfeld et al., 1995). Despite these differences researchers generally find serum and plasma BDNF levels to follow a similar pattern as, for example, evidenced by studies indicating individuals with depression have lower levels of serum and plasma BDNF than healthy control subjects (Brunoni, Lopes, \& Fregni, 2008).

Function. A primary function of neurotrophins is to promote peripheral and central nervous system (CNS) neuronal growth, differentiation, and survival (Porter \& Kaplan, 2011). Immature proBDNF has been found to prune existing neurons by binding to the p75NTR and initiating cell death (Teng et al., 2005). Mature BDNF activates cell growth, survival and regeneration through the TrkB receptor (Teng et al., 2005). BDNF's function in helping to grow new neurons is the reason for anti-depressants' effect according to the neurotrophic hypothesis of depression (Duman \& Monteggia, 2006).

Role in eating behavior. Pre-clinical studies have indicated BDNF may be implicated in eating and weight disorders. Research on animal models identified BDNF in areas of the brain known to influence eating, such as the hypothalamus, dorsal vagal complex (DVC) and paraventricular nucleus (PVN) (Bariohay et al., 2005; Wang, Bomberg, Billington, Levine, \& Kotz, 2007). BDNF and the TrkB receptor are involved in eating and weight disorders as evidenced by animal models where lack of either is associated with hyperphagia and obesity (Rios et al., 2001; Tsao et al., 2008). Mice with only one copy of the BDNF gene show increased food intake in addition to increased activity levels compared to mice who are homozygous for 
the BDNF gene (Kernie, Liebl, \& Parada, 2000). When BDNF is administered to genetically homozygous BDNF mice, decreases in food intake and body weight were seen (Kernie et al., 2000).

A study by Bariohay et al. (2005) indicated fasting rats showed decreased BDNF in the DVC compared to those fed ad libitum. When the fasting rats were refed, the levels of BDNF in the DVC were increased significantly compared to those fed ad libitum (Bariohay et al., 2005). Levels of BDNF in the hypothalamus of fasting rats did not change compared to ad libitum controls throughout the study, indicating BDNF levels in the hypothalamus were not affected by food intake while levels in the DVC were (Bariohay et al., 2005).

Because of the possible corollary of hyperphagia to clinical symptoms of binge eating (the cardinal feature of $\mathrm{BN}$ ), several investigations have begun to explore the role of BDNF and BN. Thus, the purpose of this paper is to review and summarize the findings to date comparing peripheral blood levels of BDNF in individuals with BN to healthy control (HC) subjects to inform potential directions for further study.

\section{Methods}

A systematic literature search of five databases (PubMed, CINAHL, EMBASE, PsycINFO, and MEDLINE) was conducted (see Figure 1). Search terms included eating disorders, bulimia nervosa, anorexia nervosa (because of common crossover of disorders), brain derived neurotrophic factor, and BDNF. Inclusion criteria were articles that were in English, measured BDNF levels in the serum or plasma of BN subjects, and published between 1977 and 2013. Articles on pre-clinical research (rodent studies), general or review articles, studies solely examining correlations of blood BDNF levels with psychometric scales, and genetic studies of BDNF were excluded. The initial search elicited 70 articles whose abstracts were reviewed. Of 
these, 13 full-text articles appearing to meet the inclusion criteria were examined. Subsequently, eight of the full-text articles were excluded because they did not include individuals with BN $(n=5)$ or were solely correlation in nature (e.g., focused on the relationship between BDNF and psychological variables; $n=3$ ). This resulted in the examination of five studies that assessed BDNF levels in the blood of BN subjects for inclusion in this review.

Figure 1

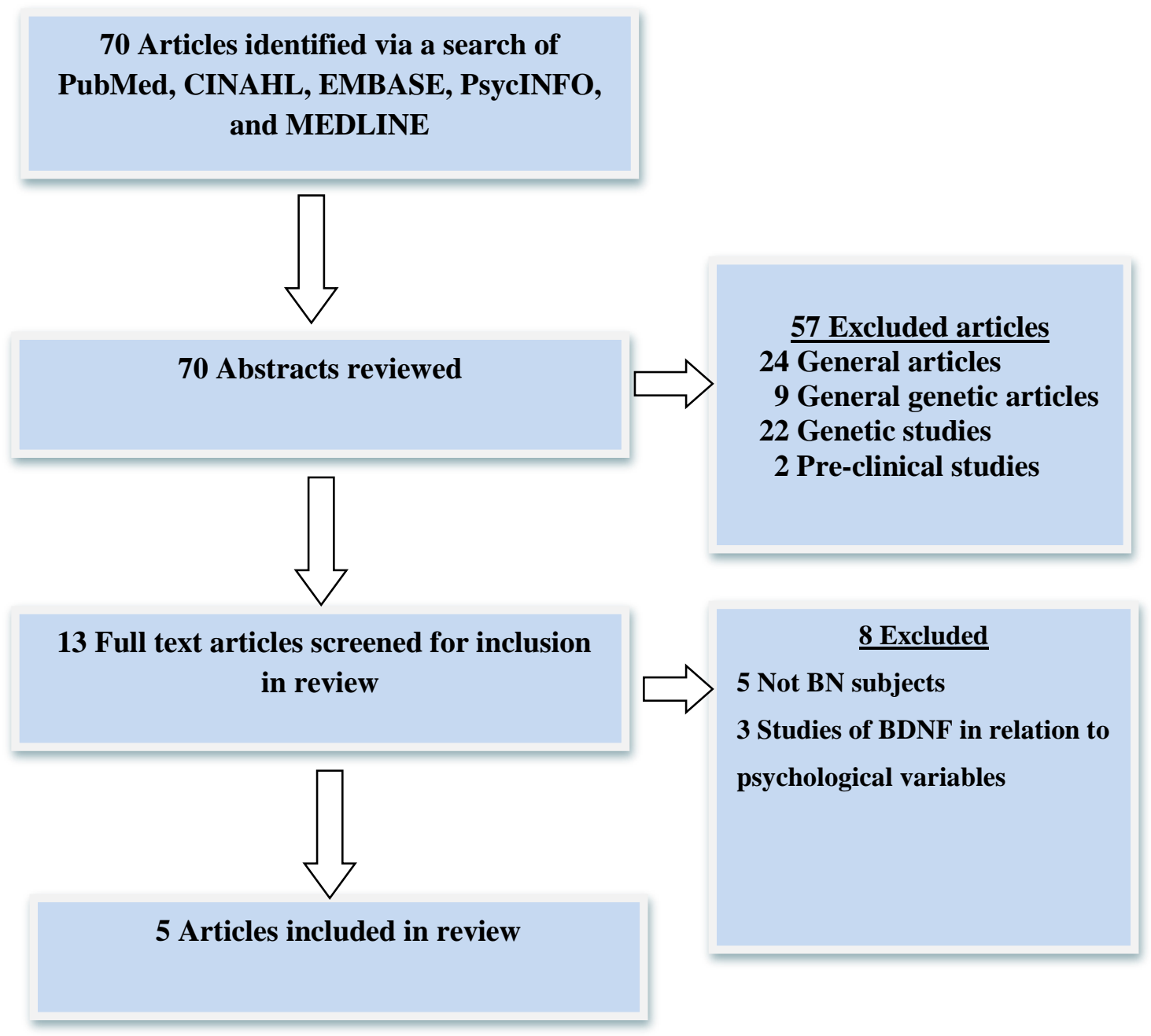

\section{Findings}

Subject Characteristics. To date, five studies have compared blood levels of BDNF in BN subjects with levels of HCs. Sample size of individuals with BN ranged from 16 to 29 
participants (Yamada, Yoshimura, Nakajima, \& Nagata, 2012; Mercader et al., 2007; Table 1). Four studies included a HC comparison group ranging from 10-24 participants (Yamada et al., 2012; Monteleone et al., 2005; Saito, Watanabe, Hashimoto, \& Saito, 2009). One additional study utilized healthy siblings of the BN participant as the control group (Mercader et al., 2007). Studies were conducted in Italy, Japan, and Spain.

\section{[INSERT TABLE 1 HERE]}

All studies included individuals who met diagnostic criteria for $\mathrm{BN}$ as defined by the Diagnostic and Statistical Manual for Mental Disorders, Fourth Edition-Text Revision (APA, 2000). Diagnosis was established in three studies (Mercader et al., 2007; Montelone et al., 2005; Yamada et al., 2012) using a version of the Structured Clinical Interview for DSM Axis I Disorders (First, Spitzer, Gibbon, \& Williams, 1995; Takahashi, Kitamua, Okano, Tomita \& Kikuchi, 2003). Two studies specified that the entire BN sample met the criteria for binge/purge type (Monteleone et al., 2005; Yamada et al., 2012). Symptom severity comparisons are complicated in that three of five studies reported duration of illness for BN (Monteleone et al., 2005; Nakazato et al., 2003; Saito et al., 2009), ranging from 3.8-6.1 years and two studies reported mean binge eating episodes (range 8.9-10.4) and vomiting (range 7.9-9.9) episodes per week (Monteleone et al., 2005; Yamada et al., 2012). With regard to comorbid conditions, Saito and colleagues (2009) indicated studying individuals free of current major depressive disorder.

The age of BN subjects was similar across studies ranging from approximately 22-26 years (Nakazato et al., 2003; Mercader et al., 2007). Control subjects' age was similar to BN subjects, ranging from approximately 21-28 years (Nakazato et al., 2003; Yamada et al., 2012). BN subjects' BMI showed a modest range across studies (Table 1) from a mean of 19.6-23.3 $\mathrm{kg} / \mathrm{m}^{2}$ (Yamada et al., 2012; Monteleone et al., 2005). In comparison, control subjects' appeared 
to have a smaller range of BMI across studies $\left(20-21.8 \mathrm{~kg} / \mathrm{m}^{2}\right)$ (Nakazato et al., 2003;

Monteleone et al., 2005).

Specimen Collection. Blood samples were collected in the morning hours (Table 2).

Two studies indicated obtaining samples following a fasting state (Monteleone et al., 2005;

Yamada et al., 2012). Blood samples consisted of both serum (Monteleone et al., 2005; Nakazato

et al., 2003; Saito et al., 2009) and plasma specimens (Mercader et al., 2007; Yamada et al.,

2012). Three studies analyzed their samples using the Emax BDNF assay by Promega (Mercader

et al., 2007; Monteleone et al., 2005; Nakazato et al., 2003) while two studies analyzed samples

with the Quantikine assay by R \& D Systems (Saito et al., 2009; Yamada et al., 2012). Two

studies reported collecting fasting samples (Monteleone et al., 2005; Yamada et al., 2012).

[INSERT TABLE 2 HERE]

Peripheral BDNF Levels. Blood values of BDNF were significantly lower than control

comparisons in three (Nakazato et al., 2003; Montelone et al., 2005; Yamada et al., 2012) of five studies (Mercader et al., 2007; Saito et al., 2009) (Table 2). Blood values of BDNF in BN participants ranged from 1.9-21.8 ng/ml using Quantikine assay and 32-49.1 ng/ml using Emax assay. For controls, BDNF blood values ranged from 6.6-26 ng/ml for Quantikine assay and 39.8-61.4 ng/ml for Emax assay. When examining those studies using non-related HCs as the comparison group, one (Saito et al., 2009) of four investigations did not show significantly lower levels of blood BDNF in the BN group.

Within the studies reviewed, several investigators examined possible clinical correlates of BDNF. This included, in BN participants, a statistically significant relationship between BMI and BDNF (r=0.63, p<0.005; Saito et al., 2009) although only observed by others (Nakazato et al., 2003). When combining subject groups (BN and controls), three studies saw a significant 
correlation between BMI and BDNF blood levels (Nakazato et al., 2003; Monteleone et al., 2005; Saito et al, 2009). Nakazato and colleagues (2003) reported a significant correlation between BDNF and depression scores (as measured by the Hamilton Depression Rating Scale [HDRS]; Hamilton, 1960) in the total sample. Others, however, did not see this relationship when using either the HDRS (Saito et al., 2009) or the Montgomery-Asberg Depression Rating (MADRS; Montgomery \& Asberg, 1979) (Monteleone et al., 2005). No relationship was observed between BDNF and age (Monteleone et al., 2005; Saito et al., 2009; Yamada et al., 2012), duration of illness (Monteleone et al., 2005; Saito et al., 2009), or symptoms of disordered eating as measured by the Eating Disorder Inventory-2 (EDI; Garner, 1991) (Monteleone et al., 2005; Saito et al., 2009) or Bulimic Investigatory Test, Edinburgh (BITE; Henderson \& Freeman, 1987) (Monteleone et al., 2005; Nakazato et al., 2003).

Finally, one study followed a subgroup of BN participants $(n=7)$ measuring plasma BDNF before and after four weeks of in-patient treatment (Yamada et al., 2012). Baseline characteristics of this subgroup were similar to the other BN subjects in the study (Yamada et al., 2012). BDNF blood levels were significantly increased from baseline to post-treatment $(1.7 \pm 0.8$ vs. $6.0 \pm 5.0 \mathrm{ng} / \mathrm{ml}, \mathrm{z}=2.4, \mathrm{p}=0.02$; mean \pm standard deviation) while mean $\mathrm{BMI}$ remained unchanged (20.3 \pm 3.1 vs. $20.3 \pm 2.9 ; \mathrm{z}=0.11, \mathrm{p}=0.92$; mean \pm standard deviation) (Yamada et al., 2012). Following in-patient treatment, plasma BDNF levels were similar in this subgroup of BN compared to control subjects (6.0 \pm 5.0 vs $6.6 \pm 6.1 \mathrm{ng} / \mathrm{ml}$ (p value not reported); mean \pm standard deviation) (Yamada et al., 2012). Additionally, following treatment, BN subjects showed a decrease in frequency of binge eating $(12.6 \pm 1.3$ vs. $0 \pm 0$ times per week; $z=2.4, p=0.02$; mean \pm standard deviation) and purging behavior, but measures of depression and anxiety remained similar to baseline ratings (Yamada et al., 2012). 


\section{Discussion}

In general, studies measuring BDNF blood values in BN subjects suggest decreased levels relative to HCs. In comparison to healthy normal-weight controls, lower BDNF blood levels also have been observed in patients with anorexia nervosa (AN) (Brandys, Kas, Van Elburg, Campbell, \& Adan, 2011; Ehrlich et al., 2009; Monteleone et al., 2004) whereas they are elevated in persons with obesity (Monteleone et al., 2004). Although three of four studies using a non-sibling healthy comparison group point to significant differences among BN and controls, the potential influence of several sample characteristics temper such conclusion at this point in time and highlight the need for further research. Saito and colleagues (2009) did not find the predicted difference in peripheral BDNF blood samples among BN individuals compared to controls. One possible explanation is that this study was the only investigation to include individuals who did not have concurrent major depressive disorder and also who were free of psychotropic medications for an extended period of time. Yet, Montelone et al. (2005) in a subgroup analysis, showed no difference in serum BDNF among those with or without (3.2 (21.3) vs. 33.9 (17.9) ng/ml) co-occurring major depression. Studies of BDNF blood levels in patients with depression have been found to be lower than controls (Sen, Duman, \& Sanacora, 2008). Similarly, studies have also shown that increases in blood BDNF is associated with antidepressant administration, although it is unclear if this is a direct correlation of the medication verses decreased symptoms (Piccinni et al., 2008; Sen et al. 2008).

The absence of a significant difference in BDNF blood values between BN individuals and controls when using siblings (Mercader et al., 2007) may point the influence of hereditability, making such differences (if they exist) potentially more difficult to observe. Interestingly this study was the only investigation to report BDNF blood levels lower for the 
control group than for the $\mathrm{BN}$ group. One possible alternative is the extent that the $\mathrm{BN}$ group was restricting food intake as study of calorie reduction in individuals who are overweight or obese has shown increases in serum BDNF (Araya, Orellana, \& Espinoza, 2008) although not observed by others (Witte, Fobker, Gellner, Knecht, \& Floel, 2009).

Further research is needed regarding the potential influence of treatment and symptom remission on blood levels of BDNF. Interestingly, Yamada and colleagues (2012) showed BDNF blood levels increased following a 4-week behavioral inpatient treatment while no significant differences were noted in BMI and measures of depression and anxiety before and after treatment. They did see a significant decrease in symptoms (binge eating and purging frequency) suggesting a possible association although this was not tested in this relatively small sample. Thus, it remains to be further elucidated if such changes are the result of treatment, decreased depressive symptoms, or decreased eating disorder psychopathology.

The role of symptom severity or state of illness (active vs. remitted) in changes in BDNF has yet to be fully examined in BN. In a study of AN patients, Ehrlich and colleagues (2009) observed that serum BDNF levels were increased in individuals recovered from AN who maintained a normal body weight for at least three months prior to study in comparison to individuals with active AN. Blood BDNF levels were higher in the recovered ANs in comparison to $\mathrm{HCs}$, approaching statistical significance $(\mathrm{p}=0.08)$, yet this relationship was not observed by others (Nakazato et al., 2009). While a relationship between body weight and peripheral BDNF levels has been reported (Lommatzsch et al., 2005), the issue of the extent to which BDNF levels are normalized or elevated following remission (e.g., from possible upregulation; Ehrlich et al., 2009) holds true for future BN studies to also examine. 
Other possible correlates of BDNF blood levels may need to be considered. In human studies, blood levels of BDNF have been found to be reduced in individuals with depression (Brunoni et al., 2008; Piccinni et al., 2008; Sen et al., 2008), who are older (Lommatzsch et al., 2005), smoke (Bhang, Choi, \& Ahn, 2010), or have increased amounts of alcohol intake (Bus et al 2011). Additionally, future studies would benefit from examination of possible neurobiological correlates such as the association with hypoleptinemia (and malnutrition) as observed in AN (Ehrlich et al., 2009).

As noted by others (Ehrlich et al., 2009), the relationship between BDNF blood levels and eating symptoms (binge episodes) and mood (depression) implicate involvement of CNS serotonin. Furthermore, the role that BDNF plays in the development and survival of neurons points to a possible mechanism for antidepressant effects on the serotonin system and possibly BDNF gene therapy (Hashimoto, Koizumi, Nakazato, Shimizu, \& Iyo, 2005). Antidepressant selective serotonin reuptake inhibitors (SSRIs) have been shown to increase BDNF gene expression and clinical response to these drugs has been postulated to be mediated by BDNF (Martinowich \& Lu, 2008). Thus, BDNF may play a role in treatment response to SSRIs in person with $\mathrm{BN}$.

Methodological variations among studies may also contribute to the findings. There was some variability in terms of whether or not subjects were studied during a fasting state and time of blood draw in the morning hours. Level of exercise prior to blood sample collection is unclear and may be useful to know in future studies as there is evidence this may increase circulating BDNF levels (Rasmussen et al., 2009). Plasma samples (vs. serum) retain more stable BDNF levels over the course of freezer storage (Bus et al., 2011; Trajkovska et al., 2007). 
Future studies are warranted to further understand the pathophysiology and role of BDNF in active $\mathrm{BN}$ and following remission. It appears that persons with $\mathrm{BN}$ have lower levels of BDNF relative to age and weight-matched controls. However, we have yet to fully understand the role of influential correlates such as depression, psychotropic medication use, smoking and drinking behaviors, and exercise. During active illness, it remains unknown to what extent changes in BDNF following treatment are related to the intervention or decreased symptoms of binge eating or purging. Furthermore, if related to treatment, the effect of various interventions remains unknown. Evidence on BDNF blood levels following remission is limited to short-term abatement of symptoms; thus, study of longer-term remission would assist in understanding the sustained effects or state vs. trait phenomenon.

It is important for psychiatric nurses to understand that these studies indicate a possible role for BDNF in the etiology of BN. Comprehension of the underlying contributors to a multifaceted etiology of the disorder is clinically important. Advances in understanding the pathophysiology of BN may point to potential avenues of clinical intervention and treatment development for psychiatric nurses. For example, a role for BDNF may be implicated in the maintenance of $\mathrm{BN}$ through cycles of binge eating and purging. As noted by previous investigators (Yamada et al., 2012), changes in eating behavior (binging, purging, and laxative use), aided by treatment interventions, may increase BDNF levels allowing the brain to adapt and heal through neuroplasticity. It is important for psychiatric nurses assessing patients with $\mathrm{BN}$ to recognize factors such as eating behaviors, mood, and substance use, which may influence an individual's baseline BDNF levels and could impact the brain's ability to handle stressors or changes in the environment. Psychiatric APRNs would benefit from understanding the relationship between BDNF and serotonin. Studies of SSRIs in depressed individuals highlight 
the relationship between BDNF and serotonin (Sen et al., 2008), which provides one possible explanation for treatment responsivity to SSRIs in persons with BN (Goldstein, Wilson, Ascroft, \& al-Banna, 1999). Thus, further elucidation of these relationships may help identify potential clinical interventions psychiatric nurses can use to influence a biological correlate that may play a role in the etiology and maintenance of the disorder or potentially serve as biomarkers for treatment responsivity.

\section{References}

American Psychiatric Association (2013). Diagnostic and statistical manual of mental disorders (5th ed.). Washington, DC: Author.

American Psychiatric Association (2000). Diagnostic and statistical manual of mental disorders ( $4^{\text {th }}$ ed., text revision). Washington, DC: Author.

Araya, A. V., Orellana, X., \& Espinoza, J. (2008). Evaluation of the effect of caloric restriction on serum BDNF in overweight and obese subjects: Preliminary evidences. Endocrine, 33, 300-304. doi: 10.1007/s12020-008-9090-х

Bariohay, B., Lebrun, B., Moyse, E., \& Jean, A. (2005). Brain-derived neurotrophic factor plays a role as an anorexigenic factor in the dorsal vagal complex. Endocrinology, 146, 56125620. doi: 10.1210/en.2005-0419

Bhang, S.Y., Choi, S.W., \& Ahn, J.H. (2010). Changes in plasma brain-derived neurotrophic factor levels in smokers after smoking cessation. Neuroscience Letters, 468, 7-11. doi: 10.1016/j.neulet.2009.10.046

Brandys, M. K., Kas, M. J., Van Elburg, A. A., Campbell, I. C., \& Adan, R. A. (2011). A metaanalysis of circulating BDNF concentrations in anorexia nervosa. The World Journal of Biological Psychiatry, 12, 444-454. doi: 10.3109/15622975.2011.562244 
Brunoni, A. R., Lopes, M., \& Fregni, F. (2008). A systematic review and meta-analysis of clinical studies on major depression and BDNF levels: Implications for the role of neuroplasticity in depression. International Journal of Neuropsychopharmacology, 11, 1169-1180. doi: 10.1017/S1461145708009309

Bus, B. A., Molendijk, M. L., Penninx, B. J., Buitelaar, J. K., Kenis, G., Prickaerts, J., . . . Voshaar, R. C. (2011). Determinants of serum brain-derived neurotrophic factor. Psychoneuroendocrinology, 36, 228-239. doi: 10.1016/j.psyneuen.2010.07.013

Conner, J. M., Lauterborn, J. C., Yan, Q., Gall, C. M., \& Varon, S. (1997). Distribution of brainderived neurotrophic factor (BDNF) protein and mRNA in the normal adult rat CNS: Evidence for anterograde axonal transport. The Journal of Neuroscience, 17, 2295-2313.

Duman, R. S., \& Monteggia, L. M. (2006). A neurotrophic model for stress-related mood disorders. Biological Psychiatry, 59, 1116-11127.

Ehrlich, S., Salbach-Andrae, H., Eckart, S., Merle, J. V., Burghardt, R., Pfeiffer, E., ... Hellweg, R. (2009). Serum brain-derived neurotrophic factor and peripheral indicators of the serotonin system in underweight and weight-recovered adolescent girls and women with anorexia nervosa. Journal of Psychiatry \& Neuroscience, 34, 323-329.

Elfving, B., Plougmann, P. H., \& Wegener, G. (2010). Detection of brain-derived neurotrophic factor (BDNF) in rat blood and brain preparations using ELISA: Pitfalls and solutions. Journal of Neuroscience Methods, 187, 73-77. doi: 10.1016/j.jneumeth.2009.12.017

First, M. B., Spitzer, R. L., Gibbon, M. \& Williams, J. B. W. (1995). Structured Clinical Interview for DSM-IV Axis I Disorders (SCID-I), Research Version. New York: Biometrics Research, New York State Psychiatric Institute.

Fujimura, H., Altar, C. A., Chen, R., Nakamura, T., Nakahashi, T., Kambayashi, J.., .. . Tandon, 
N. (2002). Brain-derived neurotrophic factor is stored in human platelets and released by agonist stimulation. Thrombosis and Haemostasis, 87, 728- 734.

Garner, D.M. (1991). Eating Disorder Inventory-2 Professional Manual. Psychological Assessment Resource: Odessa.

Goldstein, D. J., Wilson, M. G., Ascroft, R. C., \& al-Banna, M. (1999). Effectiveness of fluoxetine therapy in bulimia nervosa regardless of comorbid depression. International Journal of Eating Disorders, 25(1), 19-27.

Hamilton, M. (1960). A rating scale for depression. Journal of Neurology, Neurosurgery \& Psychiatry, 23, 56-62.

Hashimoto, K., Koizumi, H., Nakazato, M., Shimizu, E. \& Iyo, M. (2005). Role of brain-derived neurotrophic factor in eating disorders: Recent findings and it pathophysiological implications. Progress in Neuro-Psychopharmacology \& Biological Psychiatry, 29, 499504.

Henderson, M., \& Freeman, C. P. (1987). A self-rating scale for bulimia: The 'BITE.' British Journal of Psychiatry, 150, 18-24.

Huang, E. J., \& Reichardt, L. F. (2003). Trk receptors: Roles in neuronal signal transduction. Annual Review of Biochemistry, 72, 609-642. doi:

10.1146/annurev.biochem.72.121801.161629

Hudson, J. I., Hiripi, E., Pope, H. G., \& Kessler, R. C. (2007). The prevalence and correlates of eating disorders in the National Comorbidity Survey Replication. Biological Psychiatry, 61, 348-358. doi:10.1016/j.biopsych.2006.03.040

Kernie, S. G., Liebl, D. J., \& Parada, L. F. (2000). BDNF regulates eating behavior and locomotor activity in mice. The EMBO Journal, 19, 1290-1300. 
Klein, A. B., Williamson, R., Santini, M. A., Clemmensen, C., Ettrup, A., Rios, M., .. Aznar, S. (2011). Blood BDNF concentrations reflect brain-tissue BDNF levels across species. International Journal of Neuropsychopharmacology, 14, 347-353. doi: $10.1017 / \mathrm{S} 1461145710000738$

Lessmann, V., Gottmann, K., Malcangio, M. (2003). Neurotrophin secretion: Current facts and future prospects. Progress in Neurobiology, 69, 341-374. doi: 10.1016/S03010082(03)00019-4

Lewin, G. R., \& Barde, Y. A. (1996). Physiology of the neurotrophins. Annual Review of Neuroscience, 19, 289-317. doi: 10.1146/annurev.ne.19.030196.001445

Lommatzsch, M., Zingler, D., Schuhbaeck, K., Schloetcke, K., Zingler, C., Schuff-Werner, P., \& Virchow, J. C. (2005). The impact of age, weight and gender on BDNF levels in human platelets and plasma. Neurobiology of Aging, 26, 115-123.

Maisonpierre, P. C., Le Beau, M. M., Espinosa, R., Ip, N. Y., Belluscio, L., de la Monte, S. M., . . Y Yancopoulos, G. D. (1991). Human and rat brain-derived neurotrophic factor and neurotrophin-3: Gene structures, distributions, and chromosomal localizations. Genomics, 10, 558-568. doi: 10.1016/0888-7543(91)90436-I

Martinowich, K., \& Lu, B. (2008). Interaction between BDNF and serotonin: A role in mood disorders. Neuropsychopharmacology, 33, 73-83.

Mehler, P. S. (2011). Medical complications of bulimia nervosa and their treatments. International Journal of Eating Disorders, 44, 95-104. doi: 10.1002/eat.20825

Mercader, J. M., Ribases, M., Gratacos, M., Gonzalez, J. R., Bayes, M., de Cid, R., .. . Estivill, X. (2007). Altered brain-derived neurotrophic factor blood levels and gene variability are 
associated with anorexia and bulimia. Genes, Brain, and Behavior, 6, 706-716. doi:

10.1111/j.1601-183X.2007.00301.X

Monteleone, P. Tortorella, A., Martiadis, V., Serritella, C., Fuschino, A., \& Maj, M. (2004).

Opposite changes in the serum brain-derived neurotrophic factor in anorexia nervosa and obesity. Psychosomatic medicine, 66, 744-748.

Monteleone, P., Fabrazzo, M., Martiadis, V., Serritella, C., Pannuto, M., \& Maj, M. (2005).

Circulating brain-derived neurotrophic factor is decreased in women with anorexia and bulimia nervosa but not in women with binge-eating disorder: relationships to co-morbid depression, psychopathology and hormonal variables. Psychological Medicine, 35, 897905.

Montgomery, S. A., \& Asberg, M. (1979). A new depression scale designed to be sensitive to changes. British Journal of Psychiatry, 134, 382-389.

Nakahashi, T., Fujimura, H., Altar, C. A., Li, J., Kambayashi, J., Tandon, N. N., \& Sun, B. (2000). Vascular endothelial cells synthesize and secrete brain-derived neurotrophic factor. FEBS Letters, 470, 113-117. doi: 10.1016/S0014-5793(00)01302-8

Nakazato, M., Hashimoto, K., Shimizu, E., Kumakiri, C., Koizumi, H., Okamura, N., ... Iyo, M. (2003). Decreased levels of serum brain-derived neurotrophic factor in female patients with eating disorders. Biological Psychiatry, 54, 485-490. doi: 10.1016/S00063223(02)01746-8

Nakazato, M., Hashimoto, K., Shimizu, E., Niitsu, T., \& Iyo, M. (2012). Possible involvement of brain-derived neurotrophic factor in eating disorders. IUMB Life, 64, 355-361.

Nakazato, M., Tchanturia, K., Schmidt, U., Campbell, I. C., Treasure, J., Collier, D. A., . . Iyo, M. (2009). Brain-derived neurotrophic factor (BDNF) and set-shifting in currently ill and 
recovered anorexia nervosa (AN) patients. Psychological Medicine, 39, 1029-1035. doi: $10.1017 / \mathrm{S} 0033291708004108$

Pan, W., Banks, W. A., Fasold, M. B., Bluth, J., \& Kastin, A. J. (1998). Transport of brainderived neurotrophic factor across the blood-brain barrier. Neuropharmacology, 37, 1553-1561. doi: 10.1016/S0028-3908(98)00141-5

Piccinni, A., Marazziti, D., Catena, M., Domenici, L., Del Debbio, A., Bianchi, C....Dell'Osso, L. (2008). Plasma and serum brain-derived neurotrophic factor (BDNF) in depressed patients during 1 year of antidepressant treatments. Journal of Affective Disorders, 105, 279-283.

Porter, R. S., \& Kaplan, J. L. (Eds.). (2011). The Merck manual of diagnosis and therapy (19 ed.). Whitehouse Station, NJ: Merck Sharp \& Dohme Corp.

Rasmussen, P., Brassard, P., Adser, H., Pedersen, M. V., Leick, L., Hart, E., . . Pilegaard, H. (2009). Evidence for a release of brain-derived neurotrophic factor from the brain during exercise. Experimental Physiology, 94, 1062-1069. doi: 10.1113/expphysiol.2009.048512

Rios, M., Fan, G., Fekete, C., Kelly, J., Bates, B., Kuehn. R.,...Jaenisch, R. (2001). Conditional deletion of brain-derived neurotrophic factor in the postnatal brain leads to obesity and hyperactivity. Molecular Endocrinology, 15, 1748-1757.

Rosenfeld, R. D., Zeni, L., Haniu, M., Talvenheimo, J., Radka, S. F., Bennett, L., .. . Welcher, A. A. (1995). Purification and identification of brain-derived neurotrophic factor from human serum. Protein Expression and Purification, 6, 465-471. doi:

10.1006/prep. 1995.1062 
Saito, S., Watanabe, K., Hashimoto, E., \& Saito, T. (2009). Low serum BDNF and food intake regulation: A possible new explanation of the pathophysiology of eating disorders. Progress in Neuro-Psychopharmacology \& Biological Psychiatry, 33, 312-316.

Sakuma, K., \& Yamaguchi, A. (2011). The recent understanding of the neurotrophin's role in skeletal muscle adaptation. Journal of Biomedicine \& Biotechnology, 2011, 201696. doi: $10.1155 / 2011 / 201696$

Sen, S., Duman, R., \& Sanacora, G. (2008). Serum brain-derived neurotrophic factor, depression, and antidepressant medications: Meta-analyses and implications. Biological Psychiatry, 64, 527-532. doi: 10.1016/j.biopsych.2008.05.005

Takahashi, S. Kitamura, N., Okano, T., Tomita, T., \& Kikuchi, A. (2003). Japanese Version of Structured Clinical Interview for DSM-IV Axis I Disorders SCID-I. Nihon Hyoronsha, Tokyo.

Teng, H. K., Teng, K. K., Lee, R., Wright, S., Tevar, S., Almeida, R. D., ...Hempstead, B. L. (2005). ProBDNF induces neuronal apoptosis via activation of a receptor complex of p75NTR and sortilin. The Journal of Neuroscience, 25, 5455-5463. doi: 10.1523/JNEUROSCI.5123-04.2005

Trajkovska, V., Marcussen, A. B., Vinberg, M., Hartvig, P., Aznar, S., \& Knudsen, G. M. (2007). Measurements of brain-derived neurotrophic factor: Methodological aspects and demographical data. Brain Research Bulletin, 73(1-3), 143-149. doi:

10.1016/j.brainresbull.2007.03.009

Tsao, D., Thomsen, H. K., Chou, J., Stratton, J., Hagen, M., Loo, C., . . . Lin, J. C, (2008). TrkB agonists ameliorate obesity and associated metabolic conditions in mice. Endocrinology, 149, 1038-1048. doi: 10.1210/en.2007-1166 
Wang, C. F., Bomberg, E., Billington, C., Levine, A., \& Kotz, C. M. (2007). Brain-derived neurotrophic factor in the hypothalamic paraventricular nucleus reduces energy intake. American Journal of Physiology - Regulatory, Integrative and Comparative Physiology, 293, R1003-R1012. doi:10.1152/ajpregu.00011.2007

Witte, A. V., Fobker, M., Gellner, R., Knecht, S., \& Floel, A. (2009). Caloric restriction improves memory in elderly humans. Proceedings of the National Academy of Sciences of the United States of America, 106, 1255-1260. doi: 10.1073/pnas.0808587106

Yamada, H., Yoshimura, C., Nakajima, T., \& Nagata, T. (2012). Recovery of low plasma BDNF over the course of treatment among patients with bulimia nervosa. Psychiatry Research, $198,448-451$. 